\title{
Apparatus for time-resolved measurements of acoustic birefringence in particle dispersions
}

\author{
M. E. Giardini, I. Cristiani, R. Piazza, and V. Degiorgio \\ Universitá di Pavia, Dipartimento di Elettronica, 27100 Pavia, Italy
}

(Received 11 July 1994; accepted for publication 24 October 1994)

\begin{abstract}
An apparatus for time-resolved measurements of the birefringence induced in a particle suspension by an acoustic wave pulse is described. Efficient acoustic coupling is obtained by operating near the transducer resonant frequency and by matching the acoustic impedances of the cell constituents. An almost-overdamped acoustic configuration can alternatively be employed whenever a faster response is needed. Careful design of the optical setup and of the detection unit minimize diffraction and stress-birefringence parasitic effects and yields a good responsivity at fairly low acoustic intensities. A test of the apparatus on a colloidal suspension of PTFE rodlike particles is presented and discussed. (-) 1995 American Institute of Physics.
\end{abstract}

\section{INTRODUCTION}

Colloidal dispersions of nonspherical particles can be made birefringent by orienting the particles. Orientation can be obtained by application of a magnetic field (CottonMouton effect, or magnetic birefringence), an electric field (Kerr effect, or electric birefringence), or by placing the particles in a velocity gradient (Maxwell effect, or flow birefringence).

While the amplitude of these effects yields information about the strength of the specific coupling mechanisms, the dynamics of the orientation process, which can be studied, for example, by applying a field pulse, is related to the rotational diffusion of the suspended particles. Due to the strong size dependence of the rotational diffusion constant, which for spheroids scales as the cube of the particle radius, transient induced birefringence measurements are particularly promising for accurate particle sizing. We notice, however, that this latter possibility can be hardly exploited by using magnetic fields due to the very limited bandwidth of high inductance circuits, while the time-dependence of flow birefringence can be only indirectly found by analyzing the frequency dependence of the Maxwell constant. This is one of the major teasons why electric birefringence has been up till now the most popular birefringence technique. ${ }^{1}$ Furthermore, the Kerr effect in aqueous colloidal suspensions is generally large due to the marked dielectric mismatch between particles and solvent which enhances the strength of the coupling. Last but not least, Kerr effect measurements require a much simpler and cheaper setup than magnetic or flow birefringence. Electric birefringence, however, can be safely used only for weakly conducting samples to avoid the occurrence of excessive sample heating and of electrokineticelectrochemical processes which can distort the measurements and lead to sample degradation.

An alternative method which does not suffer from the above limitation, at the same time allowing one to probe birefringence transients, relies on studying the orientation of suspended particles induced by ultrasonic waves. ${ }^{2,3} \mathrm{We}$ describe in this paper an apparatus for the measurement of such an effect, known as acoustic birefringence, which exploits experimental techniques directly mutuated from time- resolved electric birefringence. The literature in acoustic birefringence is rather limited, and the orientation mechanism is only partially understood. The availability of a simple and reliable apparatus can stimulate a theoretical study of the potentiality of acoustic birefringence.

The basic experimental method consists in placing the sample in a cell which allows a good coupling with the acoustic field. Birefringence is monitored by observing the polarization changes of a laser beam linearly polarized at $45^{\circ}$ to the acoustic wave front and propagating through the cell perpendicularly to the acoustic wave. The field is applied and the induced birefringence $\Delta n$ is allowed to reach a steadystate value $\Delta n_{0}$. The field is then switched off and the birefringence decay transient is measured. As for the other induced-birefringence techniques, $\Delta n_{0}$ gives quantitative information on the coupling mechanism between the field and the particles, while the field-free relaxation transient yields the particle rotational diffusion constant. In this paper we show that, by proper design of the sample cell and detection system, the acoustic intensity necessary to obtain a detectable birefringence can be reduced to levels which are obtainable by standard low-cost laboratory equipment, being quite lower than those supplied by the high-power if amplifiers commonly employed in the past.

\section{DESCRIPTION OF THE METHOD}

\section{A. General considerations}

'I'hough observed and analyzed by Lucas $^{4}$ as early as 1938, - acoustic birefringence has been the subject of relatively few experimental and theoretical papers when compared to other birefringence effects. A review of the main theoretical aspects can be found in Ref. 2 . While acoustically induced birefringence can be observed both in simple fluids and in suspensions of colloidal particles, the origin of the effect is definitely different in the two cases.

An ultrasonic wave induces optical anisotropy in a simple fluid by mechanisms similar to those responsible of the Maxwell effect. On the contrary, the orienting torque on suspended colloidal particles is probably due to pressure differences which are generated on the surface of a particle in relative motion with respect to the fluid, a fact noticed as 
early as 1882 by Lord Rayleigh ${ }^{5}$ for the specific case of a large disk. In other words, the presence of a solid obstacle modifies the streamlines and the angular momentum associated with the flow pattern, and this will in turn generatc a reaction torque on the particle. The motion of a suspended particle will lag behind the fluid flow because of hydrodynamic inertia, so that the relative velocity between the particle and the fluid will be strongly dependent both on the particle size and on its density difference with respect to the solvent. Density mismatch very presumably plays the same role in acoustic birefringence as dielectric constant mismatch in the Kerr effect. As a consequence, it is likely that acoustic birefringence will prove more interesting for inorganic colloids rather than polymers or biological macromolecules.

A further complication in acoustic birefringence measurements is that the induced optical anisotropy can be easily masked by a much more intense acousto-optic effect. It is indeed well known that an acoustic wave induces in a fluid a refractive index modulation, which diffracts part of a light beam out of the original propagation direction. A careful experimental setup should then distinguish between the polarization change of the transmitted beam due to induced birefringence, which is seen as an intensity change after a polarizer, and the severe intensity reduction due to diffraction. Moreover, as we will see, the necessity of monitoring the transmitted intensity causes difficulties in observing the signal, since a weak optical signal must be detected in presence of a large constant background, and calls for a special design of the detection optics and electronics.

Finally, acoustic propagation presents a distinctive property which can limit the performance of an experimental setup for time-resolved measurements, namely the wide range of variation of acoustic impedances in different materials. The choice and design of all components of the apparatus must then be carefully made to ensure a good propagation pattern of the acoustic wave, avoiding the occurrence of reflection and refractions at any discontinuity.

Efficient acoustic coupling, impedance matching, deconvolution of spurious optical effects, and detection efficiency have then been the guidelines along which the experimental setup we describe has been designed.

\section{B. Cell structure}

The heart of the apparatus is the acousto-optical cell, in which the acoustic field is applied to the sample. The cell must allow a good control of the acoustic field in the sample region, where it crosses the light beam used to detect the birefringence.

In order to do so, two factors must be taken into account. First of all, at the typical acoustic frequencies used for this type of measurements, the acoustic wavelength is on the order of $0.5 \mathrm{~mm}$. To estimate the acoustic wave front emitted by a reasonably sized emitter, diffraction effects must therefore be taken into account. The cell must then allow a proper positioning of the acoustic emitter with respect to the sample. Acoustic discontinuities, which would cause reflections and refractions of the acoustic wave with consequent standing waves and unpredictable power distribution in the sample region, must be avoided.

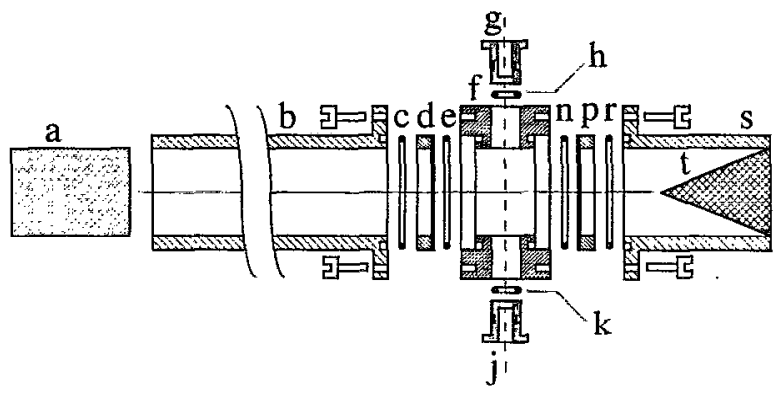

FIG. 1. The cell: (f) sample cell; (g), (j) optical windows; (d), (p) acoustic windows on mounting rings; (b), (s) coupling tubes; (a) emitter assembly; (t) absorbing cone; (c), (c), (h), (k), (n), (r) O-rings. The full line indicates the acoustic axis, the dashed line the optical axis.

Moreover, a time-resolved measurement is desired. The acoustic field must then be switched on and off very rapidly, any standing wave in the sample region would excessively slow down the field transients. The cell must then be designed to allow the application of a purely traveling acoustic wave.

Our cell is represented in Fig. 1. It consists of a stainless-steel container with two low birefringence optical windows and two thin polyethylene acoustic windows. The sample volume required is about $20 \mathrm{ml}$. The ultrasonic wave is coupled to the cell through a tube filled with a liquid whose acoustic impedance matches the acoustic impedance of the sample. The ultrasonic emitter (a PZT ceramic disk) is fixed on a pistonlike structure which can be moved into the coupling tube. This allows the sample to be positioned anywhere in the acoustic Fresnel diffraction region of the emitter. An acoustic Fresnel number $N=a^{2} /(\lambda d)$, where $a$ is the source diameter, $\lambda$ the acoustic wavelength, and $d$ the distance from the emitter, can be defined.

To work at very high $N$, the emitter can be inserted directly in the sample region of the cell; in fact, this is the working condition which gives the best wave front and the smallest sensitivity to misalignments.

The acoustic wave exiting the sample is absorbed by a PVC cone, connected to the cell by a coupling tube similar to the emitter coupler.

The absence of reflections from the acoustic windows and the absorbing cone can be verified using the PZT emitter as a standard echographic probe. To detect standing waves in the sample cell, though, a frequency resolved technique gives a much higher sensitivity. The technique is based on the fact that to any variation in the acoustic field in the cell is associated a variation in the acoustic field on the emitter surface, which can be detected as a variation of the electrical impedance of the emitter. In presence of standing waves, a measurement of the emitter impedance as a function of the acoustic frequency will then show a series of evenly spaced peaks or dips; their frequency separation will also give an accurate measurement of the distance of the two acoustic discontinuities which cause the standing wave.

\section{Ultrasonic pulse generation}

The ultrasonic pulse is generated by driving a PZT ceramic disk at a frequency corresponding to a low-order odd 


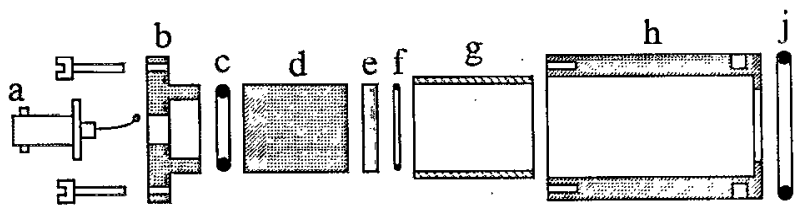

FIG. 2. Steel-backed PZT mounting: (a) BNC connector and spring contact, (b) case closure, (c) O-ring, (d) steel backing, (e) PZT disc, (f) aluminumcoatcd O-ring, $(g)$ insulating sleeve, (h) outer case, and $(\mathrm{j})$ side $\mathrm{O}$-ring.

resonance. The use of a resonant emitter enables one to reach sufficiently high acoustic intensities with low driving voltages. Any resonant structure, though, presents a transient response which will be slower as the bandwidth of the resonance is decreased. At the same time, damping the resonance to increase the bandwidth will decrease the amplitude. A tradeoff between amplitude and speed must then be found. In our apparatus two kinds of mountings have been employed, in order to exploit different working conditions.

In the first mounting, represented in Fig. 2, a $25 \mathrm{~mm}$ diameter and $2 \mathrm{~mm}$ thick Hoechst Sonox P8 disk (1 MHz nominal, 1.1 $\mathrm{MHz}$ actual fundamental thickness resonance) has been mounted on a massive steel backing. An aluminumcoated O-ring applied on the rim of the emitting surface seals the mounting and acts as a ground contact. 'The effective radiating surface is limited by an opening in the mounting to a $20 \mathrm{~mm}$ diameter circle.

This kind of mounting, having low mounting losses, does not significantly damp the resonance peaks of the ceramic disk. The emitter can therefore be driven with narrow bandwidth on the fundamental frequency or on the first two odd resonances (3.3 and 5.5 MHz). Of course, the narrow resonance peaks require an adequate frequency stabilization of the oscillator used to drive the disk. In our apparatus an HP33120A direct digital synthesis signal generator has been employed. The output of the generator is buffered using a commercial high-power integrated high-speed analog buffer/ line driver (Elantec EL 2008C). The buffer output is then sent directly to the ceramic disk (with this kind of buffer, no strict $Z$ matching of the disk to the buffer output is possible). The setup can provide peak currents up to $1.5 \mathrm{~A}$ at peak-topeak voltages up to $20 \mathrm{~V}$.

In order to obtain a wider bandwidth, a commercial echographic probe (Casoni, Milan), which is optimized in speed, can also be employed. A second mounting, which allows a $20 \mathrm{~mm}$ diameter $3.5 \mathrm{MHz}$ probe to be inserted into the coupling tube or directly into the sample cell, has therefore been constructed. With this probe, the frequency can be continuously varied from 2 to $5 \mathrm{MHz}$, with a peak efficiency around the nominal frequency. Another advantage exhibited by such a probe is that the radiating surface is flush with the mounting, thus allowing the optical beam to pass very close to it (Fig. 3).

In echographic probes, though, the high bandwidth is typically obtained by backing the emitting disk with an absorbing medium. Working on an (almost) overdamped resonance the amplitude of the acoustic wave emitted by the probe, if driven at the same conditions, is significantly

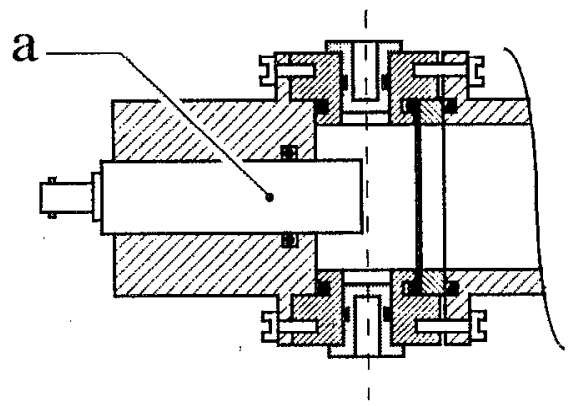

FIG. 3. Echographic probe mounting. Using the echographic probe (a) the optical beam (dashed line) can pass very close to the radiating surface.

smaller than the amplitude emitted by the steel-backed disk. In order to couple to the sample a reasonable acoustic intensity, the disk surface emits into a quarter-wave coupling layer (which works on the same principle as optical antirefiection layers). Even so; the power coupled into the cell is lower than the power which can be obtained using the first mounting, as it will be discussed in the following section.

\section{Acoustic power estimation}

In order to use the apparatus for quantitative measurements it is necessary to know the acoustic power applied to the sample.

It is, in fact, simple to determine relative powers, since the electric field applied to the ceramic disk is sufficiently small to assume that the emitting surface displacement is proportional to the applied voltage. In this situation, neglecting hysteresis and nonlinear losses; we can assume that the emitted power is proportional to the square of the applied voltage. ${ }^{6}$ This assumption will be discussed in the experimental section of this paper.

The absolute evaluation of the power is a much more challenging task. The unavailability of suitable reference standards (at least for the low acoustic powers employed in this apparatus) does not allow a standard-related calibration.

A technique which has been proposed ${ }^{7}$ is based on the analysis of the diffraction pattern of the optical beam from the phase grating induced in the sample by the acoustic wave. The main problems arising from this technique are the limited width of the optical beam compared to the grating spacing and the unknown effective grating thickness, which do not permit the use of a standard Raman-Nath grating theory; ${ }^{8}$ the alignment of the acoustic beam direction with the optical beam seems also to be excessively critical.

Notwithstanding these problems, it is possible to have a good qualitative idea of the efficiency of the ultrasonic transducer from the measurement of the diffraction pattern, thus allowing a correct selection of the operating frequency when using the narrow-bandwidth steel-backed emitter. The optical componenls necessary for this measurement have therefore been included in the apparatus. Any quantitative analysis will be postponed to future developments.

In the meanwhile the acoustic intensity $J$ coupled to the cell using the steel-backed transducer can be estimated from 
the analysis of the acoustic and piezoelectric characteristics of the materials employed:

$$
J=\frac{1}{2} \rho c\left(\frac{d_{33}}{s_{33}^{E} Z h} V\right)^{2},
$$

where $\rho, c$, and $Z$ are the density and, respectively, the sound speed and the acoustic impedance of the coupling liquid, $d_{33}$ the thickness piezoelectric charge coefficient of the ceramic disk, $s_{33}^{E}$ the short-circuit elastic compliance of the disk, $h$ the disk thickness, and $V$ the applied peak-to-peak voltage. The derivation of this relation, which assumes a fully linear operation of the transducer, an infinite impedance of the steel backing, and the absence of losses both in the transducer material and towards the mounting, can be found in Ref. 9.

Measurements of the electric power dissipated by the transducer, which can be considered as an upper limit to the radiated power, are consistent with the given formula.

The estimation of the power coupled by the commercial echographic probe cannot be made using the same technique, since the acoustic characteristics of the materials used in the probe construction are not given by the manufacturer. The measurement of the dissipated clectric power grossly overestimates the coupled acoustic power, since the probe contains a highly efficient ultrasonic absorber. The comparison of the optical diffraction pattern with the pattern generated by the steel-backed emitter indicates a significantly lower coupled power at the same driving conditions.

\section{E. Detection optics and electronics}

In order to detect the induced birefringence, we have employed with minor modifications the detection scheme traditionally used for transient electric birefringence measurements. A schematic representation of the apparatus is shown in Fig. 4.

A beam of monochromatic light emitted by a $10 \mathrm{~mW}$ $\mathrm{He}-\mathrm{Ne}$ laser is polarized by a high-extinction-ratio GlanThompson prism. The light passes through the acoustooptical cell which contains the colloidal dispersion, where it crosses the acoustic beam, forming a $45^{\circ}$ angle between the polarization direction and the acoustic propagation direction. In presence of induced birefringence, the light exiting the cell is elliptically polarized. The polarization component parallel to the original polarization is extinguished by a second Glan-Thompson prism (analyzer); the transmitted light is

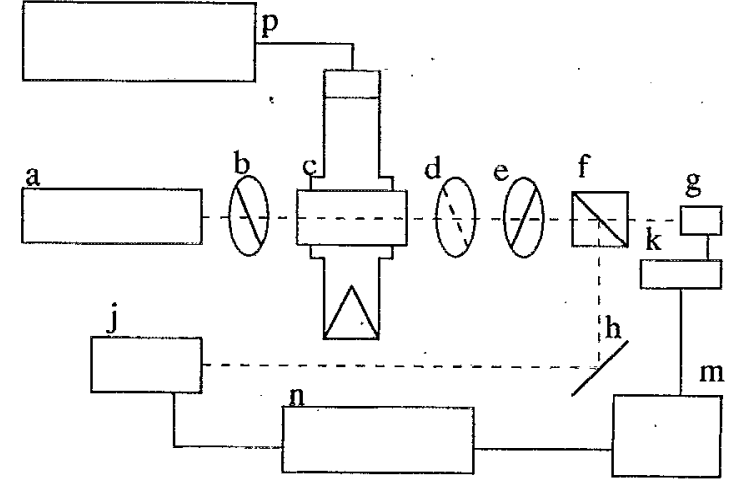

FIG. 4. Measurement setup: (a) laser, (b) polarizer, (c) cell, (p) oscillator, (d) quarter-wave plate, (e) analyzer, (f) beamsplitter, (g) detector, (k) detector filter/amplifier, (m) digitizing oscilloscope, (h) mirror, (j) CCD camera, and $(\mathrm{n})$ computer.

detected by a photodiode; the signal is then digitized and sent to a personal computer for further analysis. In order to enhance the sensitivity and to compensate any residual stress birefringence in the cell windows, ${ }^{10}$ a quarter-wave plate is inserted between the sample cell and the analyzer, and the angle between polarizer and analyzer is offset from the minimum transmittance position by an angle $\alpha$. In such conditions, the relation between the detected intensity $I$ and the birefringence $\Delta n$ is

$$
\Delta n=\frac{\lambda}{\pi \dot{d}}\left\{\arcsin \left[\sin (\alpha) \sqrt{\frac{I}{I_{\alpha}}}\right]-\alpha\right\},
$$

where $\lambda$ is the optical wavelength, $d$ the geometrical path length, and $I_{\alpha}$ the transmitted intensity in absence of birefringence. During the acoustic pulse, part of the optical power is deviated from the optical axis by diffraction effects. Employing a photodiode placed directly on the optical axis, the light spot on the photodiode active surface would then change in shape, and sensitivity inhomogeneities of the photodiode would cause a photocurrent fluctuation which would appear as a spurious signal. This signal is typically large enough to mask out any birefringence. In order to avoid such a problem, the photodiode is coupled to the cell by a wavcguide, which mixes the diffracted and transmitted beams back on the active surface, thus minimizing diffraction effects.

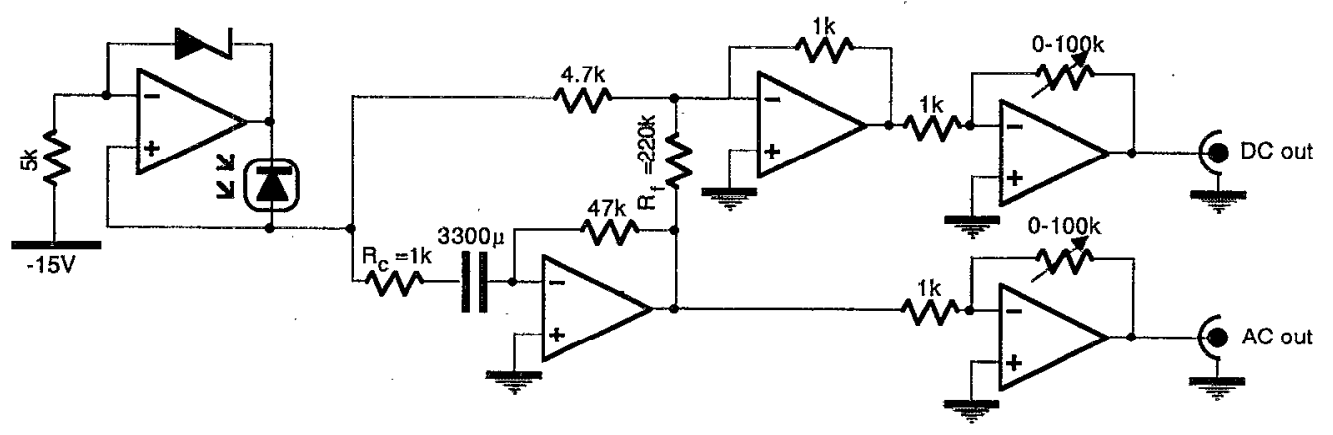

FIG. 5. Detector circuit. $R_{c}$ compensates the first ac stage; $R_{f}$ cancels the resulting signal injection in the dc path. All opamps are LF356. 
(a)

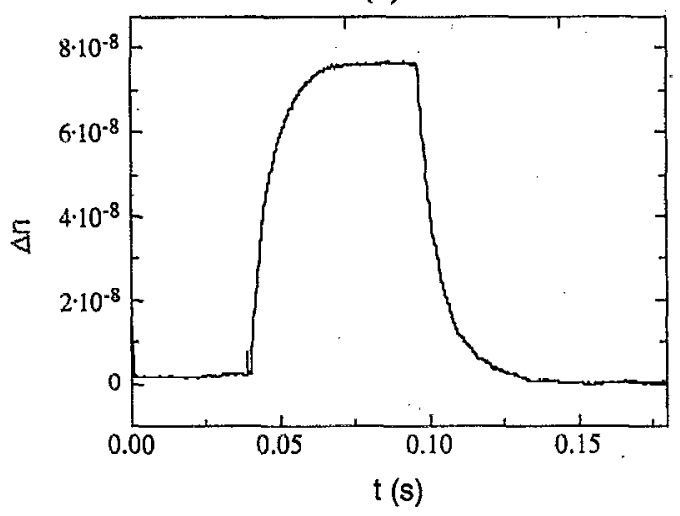

(b)

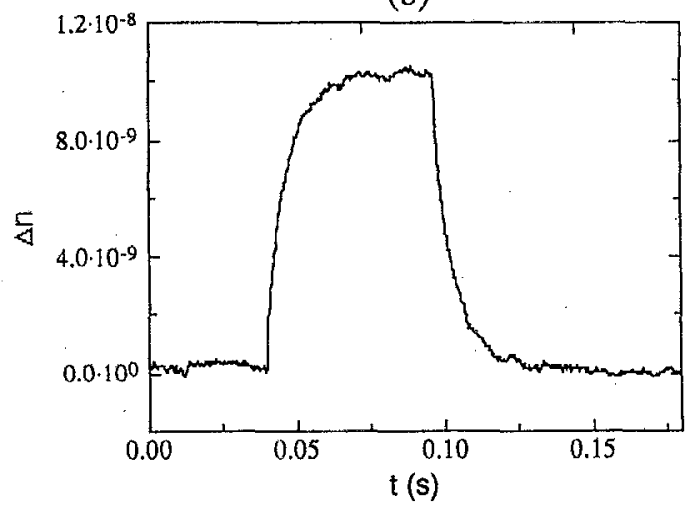

FIG. 6. Birefringence transient examples taken at an estimated acoustic intensity of (a) 0.48 and (b) $5.7 \times 10^{-2} \mathrm{~W} / \mathrm{cm}^{2}$.

In order to have access to the diffraction pattern, part of the beam is diverted from the optical axis by a beamsplitter. Since the first-order diffraction angle is very small (about $1.25 \mathrm{mrad}$ at $3 \mathrm{MHz}$ in water) the diffraction pattern is sent to a $\mathrm{CCD}$ camera after propagation in a folded structure with an equivalent length of about $2 \mathrm{~m}$. The image detected by the camera is acquired by a frame grabber for further analysis.

With this optical setup the birefringence signal detected by the photodiode appears as a small pulse over a large dc background. The signal is therefore separated into dc and ac components with a crossover filter, the two components are sent to separate amplifiers. Our filter, represented in Fig. 5, has a crossover frequency of about $0.05 \mathrm{~Hz}$.

The $\mathrm{dc}$ and ac channels are then separately digitized by a digitizing oscilloscope, and averaged over 16-256 pulses to improve the signal-to-noise ratio. The averaged signal is then set to a personal computer, which reconstructs the birefringence transient.

\section{APPARATUS PERFORMANCE}

The apparatus has been tested with a dispersion of fluorinated polymer (PTFE) particles in an aqueous solvent. From a TEM analysis, the particles can be described as polydisperse elongated spheroids with $0.35 \mu \mathrm{m}$ average major axis and axial ratio around 0.5 . The particles present an average refractive index of 1.38; the sample turbidity can then be significantly reduced by employing a refractive index matched dispersing medium consisting of a $29 \% \mathrm{w} / \mathrm{w}$ solution of urea in water. Furthermore, the particle internal structure is partially crystalline; they present therefore an intrinsic optical anisotropy. The optical behavior is uniaxial, with the optical axis coincident with the elongation direction; the difference between the ordinary and extraordinary refractive index is 0.04 . The intrinsic optical anisotropy and the elongation makes the particles well suited for birefringence measurements; an extensive characterization of similar particles by electric birefringence can be found in Ref. 11 .

The particles have been diluted to a volume fraction of $1 \%$. A small quantity of nonionic surfactant prevents aggregation of the sample.

An example of the birefringence signal is reported in Fig. 6(a). The signal has been measured using the steel- backed emitter, driven at the third resonance (3.3 MHz), working in the near field region $(N \approx 250)$. The applied intensity, estimated using Eq. (1), is $0.48 \mathrm{~W} / \mathrm{cm}^{2}$, corresponding to a peak-to-peak PZT driving voltage of $15.4 \mathrm{~V}$.

In order to extract an average rotational diffusion constant $\left\langle D_{r, a}\right\rangle$, the decay transient can be fitted with a singleexponential field-free birefringence relaxation model: ${ }^{12}$

$$
\Delta n=\Delta n_{0} e^{-6\left\langle D_{r, a}\right\rangle t},
$$

yielding $\left\langle D_{r, a}\right\rangle=22.4 \mathrm{~s}^{-1}$. Such value can be compared with the rotational diffusion constant $\left\langle D_{r, c}\right\rangle=29.8 \mathrm{~s}^{-1}$ extracted from an electric birefringence decay transient on the same sample. The (relatively small) discrepancy between the two values can be explained by the fact that when an average diffusion coefficient of a polydisperse sample is extracted from a birefringence measurement, the result depends on the relative signal contribution of each particle species. While this weight function is well known for electric birefringence measurements, no extensive analytical treatment is available for acoustic birefringence by spheroidal particles; the two average diffusion constants cannot therefore be compared directly.

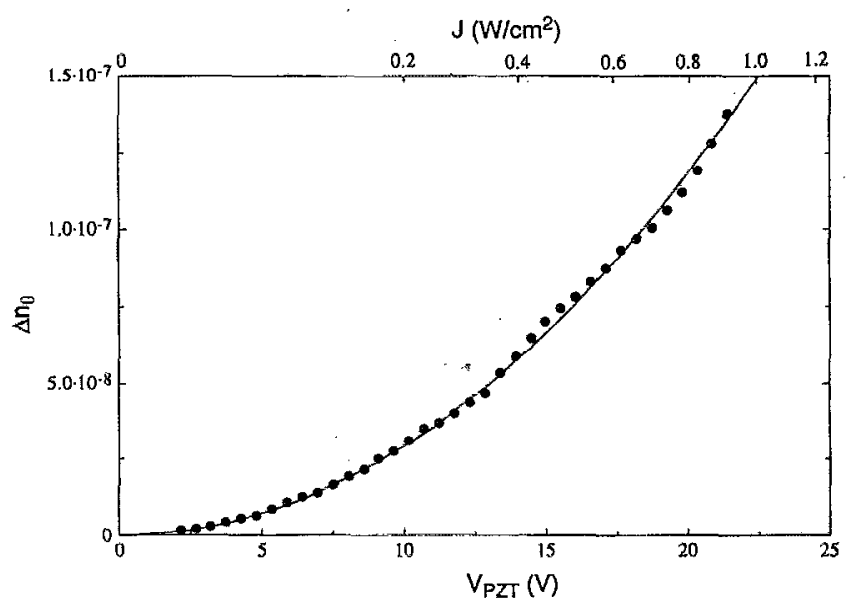

FIG. 7. Amplitude of the detected birefringence $\Delta n_{0}$ as a function of the peak-to-peak voltage $V_{\text {PZT }}$ applied to the emitter and of the estimated acoustic intensity $J$. The full line indicates a parabolic fit of the experimental data. 
In Fig. 6(b) we show a signal measured at an acoustic intensity of $5.7 \times 10^{-2} \mathrm{~W} / \mathrm{cm}^{2}$, corresponding to an emitter peak-to-peak driving voltage of $5.35 \mathrm{~V}$. It can be seen that, even at such low powers, the signal is still sufficiently clean to allow a good transient analysis.

The dependence of the amplitude $\Delta n_{0}$ of the birefringence signal from the voltage applied to the ultrasonic emitter has also been measured; the results are reported in Fig. 7 . The dependence is quadratic, yielding a linear dependence on the applied acoustic intensity [again, estimated from Eq. (1)], in agreement with previous data which can be found in literature. ${ }^{13}$ This can be an indirect suggestion that the assumption that the emitting surface displacement is proportional to the applied voltage is correct, and that the relative calibration of the emitted power can be relied upon.

It can be noted that, exploiting a resonant emitter, an accurate control of the acoustic Fresnel number, and an optical and electric detection scheme tailored on the kind of signals to be detected, this apparatus permits measurement of clean signals using an acoustic power at least two orders of magnitude lower than what required in literature for similar samples. ${ }^{14}$

\section{ACKNOWLEDGMENTS}

We wish to thank B. R. Jennings for useful advice in cell design. We also thank Montefluos for the gift of the PTFE particles, Hoechst Italia S.p.A. for the gift of the Sonox P8 disks, and S. Girometta for his assistance in the mechanical development of the cell.

${ }^{1}$ Molecular Electro-optics, edited by C. T. O'Konski (Marcel Dekker, New York, 1978).

${ }^{2}$ N. C. Hilyard, and H. G. Jerrard, J. Appl. Phys. 33, 3470 (1962).

${ }^{3}$ R. Lipeles and D. Kivelson, J. Chem. Phys. 67, 4564 (1977).

${ }^{4}$ R. Lucas, C. R. Acad. Sci. 206, 827 (1938).

${ }^{5}$ Lord Rayleigh, Philos. Mag. 14, 186 (1882).

${ }^{6} \mathrm{~J}$. Blitz, Fundamentals of Ultrasonics (Butterworths, London, 1963).

${ }^{7}$ R. Lipeles and D. Kivelson, J. Chem. Phys. 72, 6199 (1980).

${ }^{8} \mathrm{M}$. V. Berry, The Diffraction of Light by Ultrasound (Academic, London, 1966).

${ }^{9}$ T. F. Hueter and R. H. Bolt, Sonics (Wiley, New York, 1955).

${ }^{10}$ R. Piazza, V. Degiorgio, and T. Bellini, J. Opt. Soc. Am. B 3, 1642 (1986).

${ }^{11}$ T. Bellini, R. Piazza, C. Sozzi, and V. Degiorgio, Europhys. Lett. 7, 561 (1988).

${ }^{12}$ H. Watanabe and A. Morita, Adv. Chem. Phys. 56, 255 (1984).

${ }^{13}$ H. D. Ou-Yang, R. A. MacPhail, and D. Kivelson, Phys. Rev. A 33, 611 (1986).

${ }^{14}$ K. W. A. Ballinger and B. R. Jennings, Nature 282, 699 (1979). 